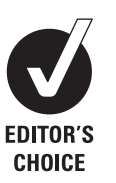

CHOICE

\title{
Prevention of sports injury l: a systematic review of applied biomechanics and physiology outcomes research
}

\author{
Kellen McBain, ${ }^{1}$ Ian Shrier, 2,3 Rebecca Shultz, ${ }^{3,4}$ Willem H Meeuwisse, 3,5 Martin Klügl, \\ Daniel Garza, ${ }^{1}$ Gordon 0 Matheson ${ }^{1,3}$
}

\begin{abstract}
- Additional appendices are published online. To view these files please visit the journal online (http://bjsm.bmj.com/ content/46/3.toc)

'Division of Sports Medicine, Department of Orthopaedic Surgery, Stanford University School of Medicine, Palo Alto, California, USA

${ }^{2}$ Centre for Clinical Epidemiology and Community Studies, Lady Davis Institute for Medical Research, Jewish General Hospital, McGill University, Montreal, Quebec, Canada

${ }^{3}$ Research Alliance in Sport and Exercise Medicine

${ }^{4}$ Human Performance Laboratory, Department of Orthopaedic Surgery, Stanford University School of Medicine, Palo Alto, California, USA ${ }^{5}$ Sport Injury Prevention Research Centre, University of Calgary, Calgary, Alberta, Canada
\end{abstract}

\section{Correspondence to}

Ian Shrier, Centre for Clinical Epidemiology and Community Studies, Lady Davis Institute for Medical Research, Jewish General Hospital, 3755 Cote Ste-Catherine Road, Montreal, OC H2T 2Y6, Canada; ian.shrier@mcgill.ca

Received 29 0ctober 2010 Accepted 2 March 2011 Published Online First 20 April 2011

\section{ABSTRACT \\ Objective To analyse published articles that used} interventions aimed at investigating biomechanical/ physiological outcomes (ie, intermediate risk factors) for sport injury prevention in order to characterise the state of the field and identify important areas not covered in the literature.

Data sources PubMed, Cinahl, Web of Science and Embase were searched using a broad search strategy. Main results Only 144 of 2525 articles retrieved by the search strategy met the inclusion criteria. Crossover study designs increased by $175 \%$ in the late 1980 s until 2005 but have declined $32 \%$ since then. Randomised controlled trial (RCT) study designs increased by $650 \%$ since the early 1980s. Protective equipment studies (61.8\% of all studies) declined by $35 \%$ since 2000 , and training studies (35.4\% of all studies) increased by $213 \%$. Equipment research studied stability devices (83.1\%) and attenuating devices (13.5\%) whereas training research studied balance and coordination (54.9\%), strength and power (43.1\%) and stretching (15.7\%). Almost all (92.1\%) studies investigated the lower extremity and $78.1 \%$ were of the joint (non-bone)-ligament type. Finally, $57.5 \%$ of the reports studied contact sports, $24.2 \%$ collision and $25.8 \%$ non-contact sports

Conclusion The decrease in crossover study design and increase in RCTs over time suggest a shift in study design for injury prevention articles. Another notable finding was the change in research focus from equipment interventions, which have been decreasing since 2000 (35\% decline), to training interventions, which have been increasing (213\% increase). Finally, there is very little research on overuse or upper extremity injuries.

\section{INTRODUCTION}

Although physical activity is associated with an overall reduction in mortality, ${ }^{1}$ morbidity ${ }^{2}{ }^{3}$ and an improved quality of life, ${ }^{45}$ it also carries an increased risk of injury and illness. ${ }^{6} 7$ The costs associated with physical activity-related injury are high. For example, in the Netherlands, every sixth hospital-treated injury is sustained during a sporting activity, ${ }^{8}$ and the indirect cost of work absence secondary to physical activity-related injury is estimated to be $\$ 525$ million annually. ${ }^{8}$ The question remains as to what extent these injuries and illnesses can be prevented.

An injury occurs when the stress applied to a tissue is greater than its ability to 'absorb' the stress acutely or chronically. This results from complex interactions between internal and external risk factors. ${ }^{9}$ Effective injury prevention programmes typically attempt to optimise the balance of applied and absorbed stress. Prevention programmes designed to decrease stress applied to a tissue can be categorised according to the cause that is being addressed. For example, the increased stress associated with 'abnormal limb alignment' can be somewhat mitigated with foot orthoses; 'poor proprioception' can be mitigated with specific training programmes and the increased stress associated with direct contact can be mitigated with appropriate equipment and padding. Furthermore, one can grossly categorise intervention strategies into three groups: (1) equipmentrelated (eg, braces, orthoses, running surfaces, clothing and footwear), (2) training (eg, muscular strength and endurance, range of motion, reaction time, proprioception) and (3) rules and regulations (which lead to a change in sport culture).

Although the overall objective of injury prevention research is to decrease injury rates, studies examining injury rates as an outcome require large sample sizes, extended periods of follow-up and are quite expensive. Therefore, much of our current basis for clinical practice comes from injury prevention research that examined intermediate outcomes, that is, risk factors for injury rather than injury itself. The purpose of this paper is to provide an overview of published articles (categorised by study design, intervention, injury location, injury type and sport) that used interventions aimed at investigating biomechanical/physiological outcomes (ie, intermediate risk factors) for sport injury prevention in order to characterise the state of the field. This should help future researchers to more easily identify the possible gaps in our knowledge base.

\section{METHODS}

We searched PubMed, Cinahl, Embase and Web of Science electronic databases (without any year restriction) using the search strategy in online appendix 1 to identify all potentially relevant English language articles. We excluded any article if the title clearly indicated it was not related to an injury prevention intervention, or only measured the physical standards of equipment (eg, impact forces absorbed by a helmet in a laboratory). Next, we reviewed all abstracts and included any original research article that reported descriptors of injury in humans (eg, valgus load); we excluded any 
article that only reported clinical outcomes such as injury risk or injury rate. Full text of each included article was obtained. A manual search of the bibliographies was performed to identify papers missed during the electronic search. We excluded articles related to performance enhancement but unrelated to injury (eg, studies using the outcome of strength for resistance training intervention were excluded, but those using the outcome of balance for resistance training were included), as well as articles where the main outcome measure was not directly related to injury prevention (eg, range of motion, bone mineral density, delayed onset muscle soreness, recovery or the effects of treatment for injury).

Using a standardised form (see table 1 for succinct summary and examples), one reviewer extracted data and a second reviewer assessed the work for errors; discrepancies were resolved by consensus with a third investigator. We extracted study information including age and sex of participants, injury history (first-time, recurrent), study design, type of intervention, location of injury, type of injury and sport according to the classifications below.

\section{Age}

We classified articles according to the age range mentioned in the article (an article could have multiple classifications) using the following categories: $(1)<18$, (2) $18-24$ and (3) $\geq 25$. If the age range was not mentioned, we estimated age ranges based on logical assumptions (eg, a study on NCAA Division I athletes and infantry personnel was classified as 18-24; a study on senior players mean (SD) age of 25 (1.5) was classified as both $18-24$ and $\geq 25$ ).

Table 1 Succinct summary of data abstraction form providing study inclusion and exclusion criteria and categories used during the data abstraction process

\begin{tabular}{ll}
\hline Item & Terms \\
\hline Inclusion criteria & Language:English \\
& Topic:Injury prevention \\
& Population:Human \\
& Outcome:Physiological or biomechanical measure \\
& Outcome:Injury rates, injury risk, restricted \\
& to performance enhancement \\
Exclusion criteria & <18; 18-24; $\geq 25$ \\
& Recurrent:Specifically mentions recurrence, \\
Age categories* & re-injury, second injury \\
Injury history* & First-time:All other articles \\
& Randomised controlled trial; crossover; \\
& pre-post; cohort; case-series, cross-sectional \\
Study design & Equipment:Stability device (eg, athletic tape, show \\
type, wrist guards); attenuation device (eg, shock & absorbing insoles, safety balls, impact bases); other \\
Intervention type* & (eg, head/face protectors, playing surface, thigh \\
protector) & Training:Balance/coordination (eg, wobble board, \\
balance foam); strength/power (eg, plyometric, \\
eccentric); stretching (eg, stretching effects on \\
position sense, reflex activity); other (eg, proper \\
landing technique, supervised training feedback \\
instructions, verbal instruction) \\
Head/neck/spine; trunk; upper extremity; lower \\
extremity; other \\
Central-peripheral nervous system (eg, concussion, \\
nerve, spinal cord compression, brain); \\
contusion-laceration-abrasion; fracture-bone stress; \\
joint (non-bone)-ligament (eg, sprains, cartilage, \\
patello-femoral syndrome); muscle-tendon; other \\
See appendix 2 \\
\hline
\end{tabular}

*Where articles included more than one category, we used all relevant categories.

\section{Injury history}

We classified articles as 'recurrent' injuries if the article specifically mentioned recurrence, re-injury or second injury, and all others as first-time injury (eg, 'healthy participants' or 'no history of injury within 6 months').

\section{Study design}

We classified a study as a randomised controlled trial (RCT) if participants were randomised into an intervention and a comparison (eg, placebo or control) group. We classified a study as a crossover design if all subjects received all treatments in random order (when possible) and as a pre-post design if the main comparison group consisted of participants before and after a single intervention. Studies in which the researcher did not apply the intervention (observational) and those that compared groups receiving different types of exposures (eg, taping vs bracing) were considered as a cohort design (this included studies with pre-post measures as long as the main comparison of interest was between groups). We considered studies that simply followed participants over time as case series, and articles that measured exposure and outcome at the same time as cross-sectional. For studies that included multiple types of analyses within one paper, we classified them based on the most important outcome.

\section{Intervention type}

We classified an article as follows: (1) 'equipment' if it examined protective devices, (2) 'training' if the objective was to induce a neuromuscular adaptation or (3) 'other' (eg, nutritional supplementation). We further classified equipment and training interventions into several categories. Equipment articles were categorised as follows: (1.1) stability device, (1.2) attenuating device and (1.3) other. Training articles were categorised as follows: (2.1) balance/coordination, (2.2) strength/power, (2.3) stretching and (2.4) other. In cases where the training programmes could be categorised into more than one group, we used all of the relevant categories (ie, studies that used a wobble board and plyometric exercises were categorised as both balance/coordination and strength/ power).

\section{Injury locations and injury type}

We used categories similar to previous publications. ${ }^{10} 11$ In brief, locations included head/neck/spine, trunk, upper extremity, lower extremity and other. Injury types included central-peripheral nervous systems, contusion-lacerationabrasion, fracture-bone stress, joint (non-bone)-ligament, muscle-tendon and other.

\section{Sport type}

We extracted each sport mentioned and grouped them for analysis according to online appendix 2. Collision sports were grouped together and included both alpine skiing and snowboarding because of the risk of high-speed falls/collision. Sports that involved very little risk of contact were grouped together (eg, swimming, squash), as were sports where the contact occurs frequently but collision is rare (eg, basketball, baseball). Military studies were removed from this categorisation.

\section{RESULTS}

The search strategy is illustrated in figure 1. Our search yielded 2525 articles, and 330 were considered relevant after the title 
and abstract search. The bibliography search yielded an additional 71 papers (total $n=401$ ) that were potentially appropriate. Of these, after reading the full text, 118 were excluded because they did not meet our inclusion criteria and 139 articles measured clinical outcomes instead of intermediate outcomes. We reviewed the remaining 144 papers in detail.

\section{Population demographics (age, sex, injury history)}

Among the articles that included age ( $n=134 / 144), 9.7 \%$ only included participants aged $\leq 18,25.4 \%$ only included participants aged $18-24,4.5 \%$ only included participants aged $\geq 25$ and more than $50 \%$ included participants across two or more age group categories. With respect to sex $(n=144), 32.6 \%$ of the articles had exclusively male participants, $20.8 \%$ had exclusively female participants and $46.5 \%$ included both male and female participants. Of the 123 articles that clearly mentioned the participants' injury history, $72.4 \%$ focused on preventing first-time injuries and $27.6 \%$ included recurrent injury prevention programmes (either alone or in combination with new injuries).

\section{Study design}

Overall, $57.6 \%$ of the injury prevention programmes used a crossover study design, $22.9 \%$ an RCT design, $11.1 \%$ a pre-post design and $8.3 \%$ used either a cohort or cross-sectional study design. The number of articles published per 5 -year interval stratified by study design is shown in figure 2. Historically, the most common study design for biomechanical injury prevention programme studies was the crossover design. Before $2005,71 \%$ of the articles used this type of design. From 2005 to 2010, RCTs have increased and crossover studies have decreased such that the number has been about equal over this time period.

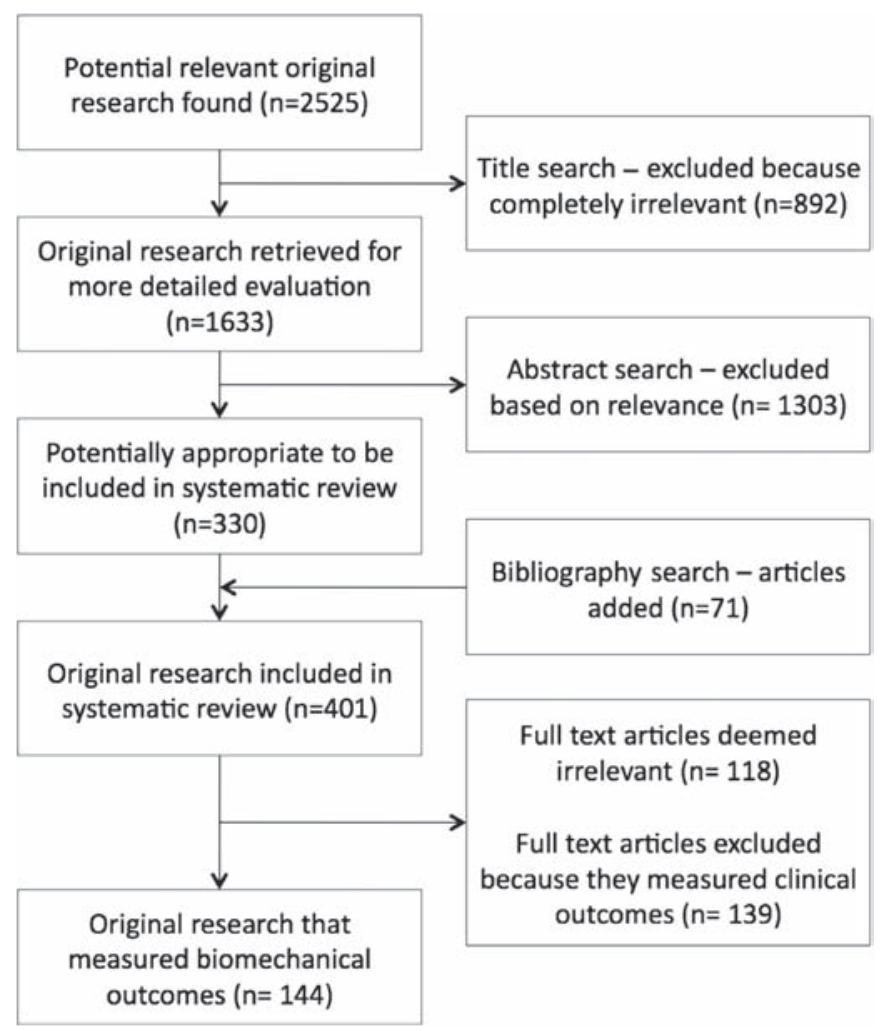

Figure 1 Process used for inclusion and exclusion of articles. In the end, 144 articles were selected for analysis.

\section{Intervention type}

Figure 3 illustrates the number of studies published over time stratified by the type of intervention. Protective equipment was the focus of $61.8 \%$ of the included articles $(n=89)$, training was the focus of $35.4 \%(n=51)$, and there were four in the other category (one on bicycle saddle angle, two on supplementation and one on landing conditions).

Figure 4 further details the types of equipment (figure 4A) and training-related (figure $4 \mathrm{~B}$ ) research. Within the equipment studies, $83.1 \%$ used a stability device. Research into stability devices peaked between 1996 and 2000 and has since decreased by approximately $65 \%$. The total number of articles in either the 'attenuating device' or 'other' group was $\leq 5$ during any 5 -year period. For training studies, balance and coordination interventions began to increase in the early 1990s, whereas articles related to strength and power or stretching began to increase in the early 2000s. The total number of articles in any one group remained low over any 5 -year period (ie, always $\leq 15$ ).

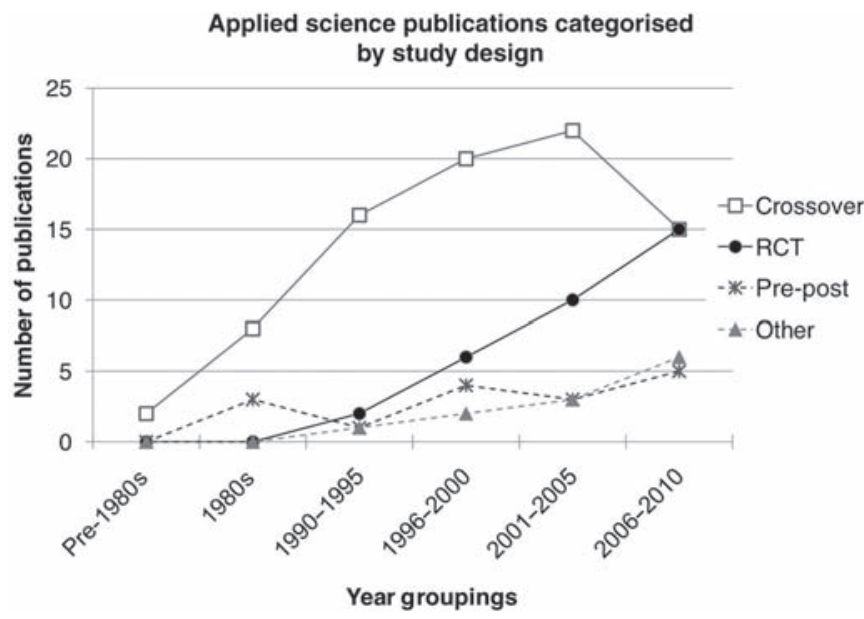

Figure 2 The data are grouped into 5-year intervals to illustrate trends over time. Publications were categorised as crossover studies, randomised controlled trials (RCTs), pre-post (measured before and after intervention) and other (cohort or cross-sectional).

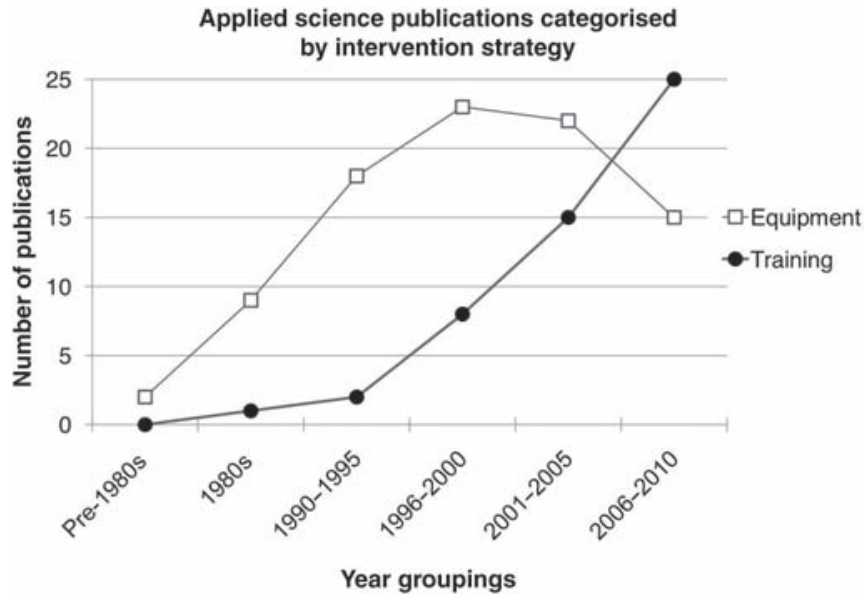

Figure 3 Equipment studies are those using protective devices for interventions and include footwear, training and running surfaces, braces and mechanical devices. Training studies are those where the interventions were designed to induce a neuromuscular adaptation. 


\section{Injury location and type}

Of the 139 articles that mentioned injury location, 92.1\% investigated lower extremity injuries and of those, $78.1 \%$ were of the joint (non-bone)-ligament type. Of the remaining articles, four were related to the head/neck/spine, one was related to the trunk and two were related to the upper extremity.

\section{Sports}

Figure 5 provides additional details about articles with respect to sport and injury site. Of the 66 articles that mentioned sport, $57.5 \%$ included contact sports, $24.2 \%$ collision sports and $25.8 \%$ non-contact sports (the total is $>100 \%$ because some studies included more than one type of sport). The vast majority of articles targeted the lower extremities ( $n=59,89.4 \%$ ) and were designed for contact sport athletes ( $n=37,62.7 \%$ ). These studies almost exclusively studied risk factors for joint (non-bone)-ligament injuries.

\section{DISCUSSION}

Our review yielded three significant findings. Given the importance of sport injury prevention research, the most surprising finding was that only 144 publications were found where interventions were designed to identify factors that would lead to a reduction in injury risk. Second, whereas earlier studies used
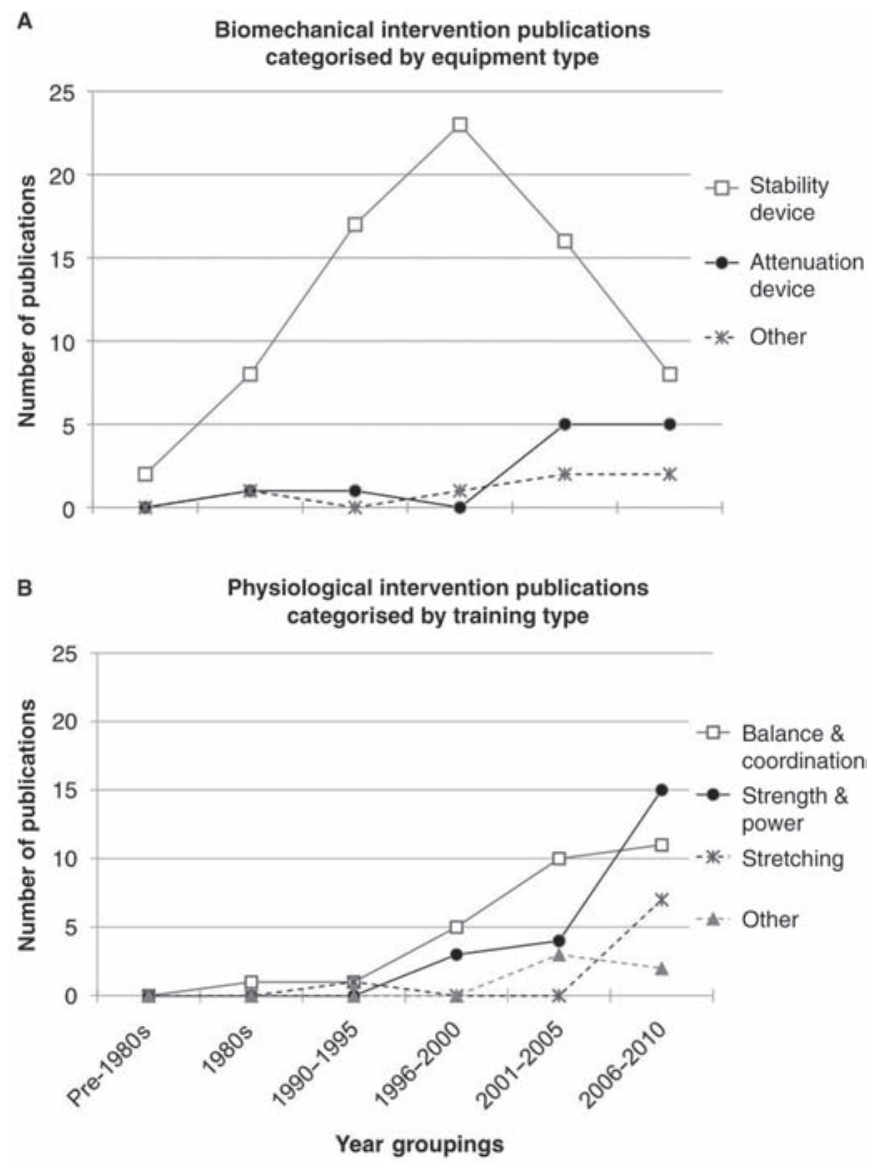

Figure 4 Equipment interventions $(A)$ were subcategorised into stability devices (eg, brace, tape, orthosis); attenuating devices (eg, shock absorbing insoles) or other (eg, head/face protectors or playing surface). Training interventions (B) were subcategorised into balance and coordination (eg, wobble board); strength and power (eg, resistance training); stretching (eg, effects on position sense) or other (eg, supervised training, technique modification). the crossover design to study equipment-related factors, the recent trend is towards studying training-related factors in randomised controlled study designs. Finally, the vast majority of studies examined risk factors for lower extremity injuries in contact sports.

The recent decrease in crossover studies in favour of RCTs (figure 2) is interesting given that crossover studies are generally considered a stronger study design when appropriate. Whereas an RCT compares an intervention group to a control group, crossover designs allow the patient to act as his or her own control, which can reduce bias. However, it is generally recommended that subjects need to return to baseline before the second intervention is applied. Therefore, crossover designs are often used when comparing stability or attenuating devices (which represented the majority of studies before 2000 as seen in figure 3), whereas RCT designs are often used for training interventions (which represent a rapidly increasing proportion of studies since 2000) because it is inappropriate for researchers to 'detrain' subjects after an injury prevention training intervention.

Figure 3 shows that equipment studies have been decreasing since 2000 whereas training studies have been increasing. These results demonstrate that the intermediate outcome research follows the more general pattern of injury prevention research recently demonstrated by Klügl et al. ${ }^{12}$ There are two possible general reasons for this shift from a more passive form of prevention (equipment) to an active form (training). First, the rapid increase in equipment studies between 1996 and 2000 might reflect the vast amount of research done on ankle and knee stability devices when technology began to allow more precise measurements that were not previously possible. ${ }^{13}$ The recent decline might simply reflect the fact that many of the questions have now been adequately answered with the current technology. Should truly innovative protective equipment be developed, one would expect another rapid increase in these types of studies. Alternatively, Klügl et al ${ }^{12}$ suggested that equipment-related research is often funded by industry in order to test their products. Once a type of equipment is selling well, industry may simply invest research and development funds into updating and marketing these products, without investing more funds into efficacy or effectiveness research. Regardless of the reason for the decrease in equipment-related research, such a decline would naturally result in researchers looking at other interventions, such as training studies. The finding that

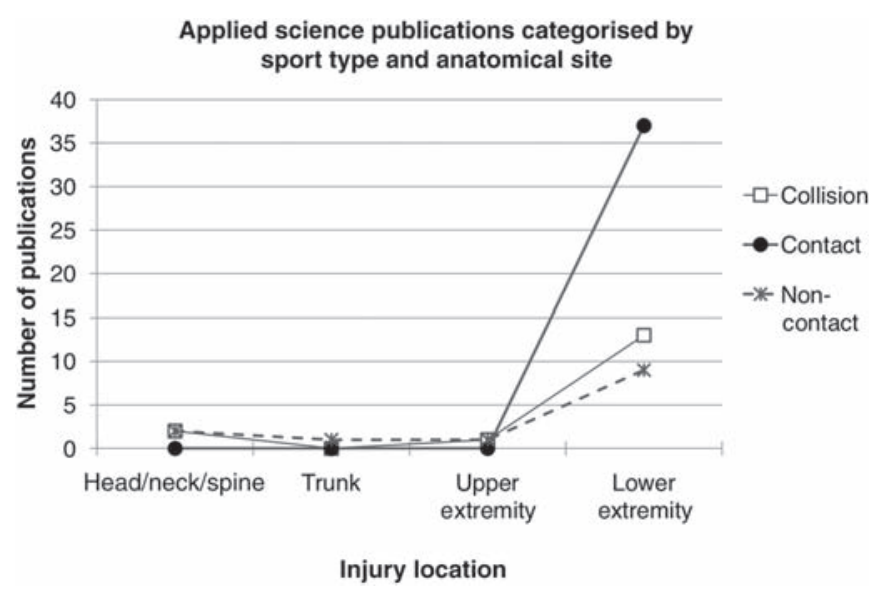

Figure 5 Sport categories include collision (eg, North American football), contact (eg, basketball, handball) and non-contact (eg, swimming). 


\section{What is already known}

Overall, injury prevention research on reducing risk factors (intermediate outcomes) has been increasing.

\section{What this study adds}

- An overview of the changing trends over time for injury prevention research that examines the effect of interventions on risk factors for injury.

- Identification of gaps in the 'risk' injury prevention literature, especially the very low number of studies examining prevention for non-contact and upper extremity injuries.

training studies are increasing proportionately in all three categories is likely a reflection of the paucity of data before $2000(\leq 5$ articles for any subcategory of training in any period), and our lack of understanding as to which measures are most related to an actual decrease in injury risk and rates.

We also found that $92.1 \%$ of articles specifically studying an injury location were restricted to the lower extremity, and $82 \%$ of articles studied collision or contact sports (figure 5). The lack of research into intermediate outcomes in non-contact sports (ie, where more overuse injuries are expected) and upper extremity injuries represents an important gap in our knowledge. For example, proprioception is considered as an important aspect of prevention in the lower extremity based on previous research. ${ }^{14}{ }^{15}$ Although some clinicians incorporate proprioception training for the upper extremity as well, ${ }^{16}{ }^{17}$ our findings suggest that the effectiveness of these programmes has not been sufficiently documented.

Finally, there is usually a delay between a landmark article on a topic and subsequent large increase in research in that field. Ekstrand ${ }^{18}$ published the first injury prevention programme that focused on a training protocol as the intervention. About a decade later, we see a sharp spike in the number of training programmes (see figure 3). Tropp et a $1^{19}$ published the first balance and coordination training intervention. About a decade later, we see a spike in the number of published balance and coordination training interventions (see figure 4B). The expansion of research related to stretching and prevention occurred 10 years after Shrier ${ }^{20}$ published a review article that suggested stretching before exercise is unlikely to reduce the risk of injury. These delayed results are not specific to sport medicine and have been reported in other areas of medical research. ${ }^{21}$ Although there are many reasons for delays between research and subsequent action, ${ }^{21}$ at least part of the reason in this context is the time required to write a grant, obtain funding, carry out the study and then publish the results.

\section{LIMITATIONS}

Although we used a broad search strategy over several electronic databases and scanned the bibliographies of included papers, we may have missed some articles. In order to maximise efficiency, we used a second reviewer to validate all data extraction rather than rely on independent data abstraction. Finally, we limited our study to English language publications and would have missed articles in other languages.
In summary, our findings suggest that research examining intermediate outcomes for equipment-related interventions is declining and training-related interventions are increasing. Almost all research is focused on contact or collision sports and injuries of the lower extremity. There is almost no injury prevention research for intermediate outcomes for the upper extremity or overuse injuries, and few injury prevention studies related to head, neck, trunk and spine injuries. Moreover, the fact that only 144 publications of this nature exist suggests that despite many articles published in the context of injury prevention in sport, few are actually designed to study biomechanical and physiological interventions for sport injury.

Acknowledgements This publication represents work done by members of RAISEM (Research Alliance in Sport and Exercise Medicine). RAISEM undertakes group research projects on sport injury prevention, exercise medicine, clinical treatment and return to play. The members of the group include clinicians, content experts, epidemiologists, biomechanists and physiologists, with associated members collaborating on specific projects.

Provenance and peer review Not commissioned; externally peer reviewed.

\section{REFERENCES}

1. Blair SN. Effects of physical activity on cardiovascular disease mortality independent of risk factors. Physical Activity and Cardiovascular Health: A National Consensus. Champaign, IL: Human Kinetics 1997:127-36.

2. Siscovick DS, LaPorte RE, Newman JM. The disease-specific benefits and risks of physical activity and exercise. Public Health Rep 1985;100:180-8.

3. Sothern MS, Loftin M, Suskind RM, et al. The health benefits of physical activity in children and adolescents: implications for chronic disease prevention. Eur J Pediatr 1999;158:271-4.

4. Dias RC, Dias JM, Ramos LR. Impact of an exercise and walking protocol on quality of life for elderly people with OA of the knee. Physiother Res Int 2003;8:121-30.

5. Oka RK, De Marco T, Haskell WL, et al. Impact of a home-based walking and resistance training program on quality of life in patients with heart failure. Am J Cardiol 2000;85:365-9.

6. Kra A. Sport-related injuries in an emergency department (prospective study with 310 cases). J Traumatol Sport 2008;24:204-8.

7. Gabbe BJ, Finch CF, Cameron PA, et al. Incidence of serious injury and death during sport and recreation activities in Victoria, Australia. Br J Sports Med 2005;39:573-7.

8. Schmikli SL, Backx FJ, Kemler HJ, et al. National survey on sports injuries in the Netherlands: target populations for sports injury prevention programs. Clin J Sport Med 2009;19:101-6.

9. Meeuwisse WH. Predictability of sports injuries. What is the epidemiological evidence? Sports Med 1991;12:8-15.

10. Shrier I, Meeuwisse WH, Matheson GO, et al. Injury patterns and injury rates in the circus arts: an analysis of 5 years of data from Cirque du Soleil. Am J Sports Med 2009;37:1143-9.

11. Fuller CW, Raftery M, Readhead C, et al. Impact of the International Rugby Board's experimental law variations on the incidence and nature of match injuries in southern hemisphere professional rugby union. S Afr Med J 2009;99:232-7.

12. Klügl M, Shrier I, McBain K, et al. The prevention of sport injury: an analysis of 12,000 published manuscripts. Clin J Sport Med 2010;20:407-12.

13. Sutherland DH. The evolution of clinical gait analysis. Part II kinematics. Gait Posture 2002;16:159-79.

14. Emery CA, Cassidy JD, Klassen TP, et al. Effectiveness of a home-based balancetraining program in reducing sports-related injuries among healthy adolescents: a cluster randomized controlled trial. CMAJ 2005;172:749-54.

15. Pánics G, Tállay A, Pavlik A, et al. Effect of proprioception training on knee joint position sense in female team handball players. Br J Sports Med 2008;42:472-6.

16. Partin NB, Stone JA, Ryan EJ, et al. Upper extremity proprioceptive training. J Athl Train 1994;29:15-18.

17. Lust KR, Sandrey MA, Bulger SM, et al. The effects of 6-week training programs on throwing accuracy, proprioception, and core endurance in baseball. J Sport Rehabil 2009;18:407-26.

18. Ekstrand J. A training program for the prevention of injuries to reduce soccer injuries by 75 per cent. Nord Med 1982;97:164-5.

19. Tropp H, Askling C, Gillquist J. Prevention of ankle sprains. Am J Sports Med 1985;13:259-62.

20. Shrier I. Stretching before exercise does not reduce the risk of local muscle injury: a critical review of the clinical and basic science literature. Clin J Sport Med 1999:9:221-7.

21. Sussman S, Valente TW, Rohrbach LA, et al. Translation in the health professions: converting science into action. Eval Health Prof 2006;29:7-32. 